\title{
El giro de la investigación universitaria hacia la acción pública: el debate sobre los Centros de Pensamiento en Colombia
}

\author{
Lina-Rocío Medina-Muñoz
}

\section{RESUMEN}

Este artículo examina los debates que sostienen los miembros de la comunidad académica colombiana con respecto a la reorganización de la investigación universitaria en Centros de Pensamiento, con el propósito de categorizar las tensiones que han emergido como resultado de este proceso y establecer qué aspectos de la actual organización deberían ser transformados. Para analizar estos debates se diseñó una aproximación metodológica cualitativa que atiende a los principales conceptos propuestos tanto por la sociología de la ciencia como por los estudios de redes. En primer lugar, se realizó una breve descripción del marco normativo que reestructuró el Sistema Nacional de Ciencia y Tecnología a partir de los años noventa. Luego, se caracterizaron las tensiones surgidas alrededor de los cambios organizativos, documentándolas mediante entrevistas semiestructuradas y ejercicios de observación etnográfica. Finalmente, se discutieron los obstáculos que deberían ser removidos por una reestructuración organizativa, a fin de que los productos de investigación tengan mayor incidencia social. Este trabajo concluye que la participación de los académicos en equipos de investigación heterogéneos se percibe ahora como un factor diferencial para ampliar el impacto del conocimiento universitario en la esfera pública.

Palabras clave: investigación científica, Centros de Pensamiento, política de investigación, asociacionismo, multidisciplinariedad, Colombia. 
O giro da pesquisa universitária em direção à ação pública:

o debate sobre os Centros de Pensamento na Colômbia

\title{
RESUMO
}

Este artigo examina os debates que os membros da comunidade acadêmica colombiana mantêm a respeito da reorganização da pesquisa universitária em Centros de Pensamento, com o propósito de categorizar as tensões que têm emergido como resultado deste processo e estabelecer que aspetos da atual organização deveriam ser transformados. Para analisar estes debates planejou-se uma aproximação metodológica qualitativa que atende aos principais conceitos propostos tanto pela sociologia da ciência como pelos estudos de redes. Em primeiro lugar, se realizou uma breve descrição do marco normativo que reestruturou o Sistema Nacional de Ciência e Tecnologia a partir dos anos noventa. Logo, se caracterizaram as tenções surgidas ao redor das mudanças organizativas, documentando-as mediante entrevistas semiestruturadas e exercícios de observação etnográfica. Finalmente, se discutiram os obstáculos que deveriam ser removidos por uma reestruturação organizativa, a fim de que os produtos de pesquisa tenham maior incidência social. Este trabalho conclui que a participação dos acadêmicos em equipes de pesquisa heterogêneas se percebe agora como um fator diferencial para ampliar o impacto do conhecimento universitário na esfera pública.

Palavras chave: pesquisa cientifica, Centros de Pensamento, política de pesquisa, associacionismo, multidisciplinaridade, Colômbia.

The shift of university research towards public action: the debate about Think Tanks in Colombia

\begin{abstract}
This article examines the debates held by members of the Colombian academic community regarding the reorganization of university research into Think Tanks, with the purpose of categorizing the tensions that have emerged as a result of this process and to establish which aspects of the current organization should be transformed. In order to analyze these debates, a qualitative methodological approach was designed that takes into account the main concepts proposed by both the sociology of science and network studies. First, a brief description was carried out of the regulatory framework that restructured the National Science and Technology System since the 1990s. Then, the authors characterize the tensions that arose around the organizational changes, documenting them through semi-structured interviews and ethnographic observation exercises. Finally, they discuss the obstacles that should be removed by organizational restructuring in order for research products to have greater social impact. This paper concludes that the participation of academics in heterogeneous research teams is now perceived as a differential factor to broaden the impact of university knowledge in the public sphere.
\end{abstract}

Key words: scientific research, think tanks, research policy, associationism, multidisciplinarity, Columbia.

Recepción: 10/04/20. Aprobación: 15/12/20 


\section{Introducción}

La centralidad de la investigación en la presente etapa de desarrollo del conocimiento tecnocientífico ha convertido su gobernanza en un asunto prioritario para diferentes actores sociales. Si en el pasado hubo confianza en que la propia dinámica del sistema científico llevaría a la producción de conocimiento útil, ahora se considera que todos los aspectos de la investigación deben ser administrados y gestionados para que respondan eficazmente a diferentes demandas. $\mathrm{Al}$ igual que la comunidad académica, cuya ambición es producir conocimientos que ayuden a generar innovaciones adicionales, los gobiernos esperan que la investigación pueda crear soluciones eficaces para atender los problemas sociales. La industria, por su parte, busca implementar conocimientos que se transformen en ventajas comparativas y, en general, la sociedad confía en que los investigadores contribuyan tanto a explicar sus problemas como a incrementar su bienestar.

La convergencia de estos intereses puso de manifiesto que era necesario hacer cambios organizativos para crear una infraestructura científica y tecnológica más competente y fuerte. En este contexto, la colaboración científica se ha ubicado en primer lugar entre una serie de factores que pueden ayudar a explotar el potencial de la ciencia para renovarse y sacar provecho de sus transformaciones. La conformación de espacios inter y multidisciplinares se ha convertido en una meta para los científicos que, desde la industria o desde centros de investigación paralelos a las universidades, aspiran a conseguir un mayor rendimiento de las actividades investigativas. Esta dinámica ha provocado no sólo una diversificación de los espacios y las formas en que se organiza la investigación, sino una densificación de las redes académicas nacionales y globales. Sin embargo, esta tendencia también busca modelar patrones de organización más diversificados en las universidades, para orientar tanto la investigación como la trasferencia tecnológica y la enseñanza. Aun así, este fenómeno ha sido escasamente estudiado por la literatura reciente, la cual se ha concentrado en el análisis de las redes internacionales y la productividad científica global.

En este artículo, por lo tanto, se examinarán los debates surgidos entre los académicos colombianos a raíz de la estructuración de la investigación universitaria en Centros de Pensamiento (CP) o Think Tanks, con el propósito de categorizar dichas tensiones y establecer sobre qué aspectos pretende influir la reorganización de esta función universitaria. En primer lugar, se precisará qué clase de estrategias organizativas han sido creadas recientemente para estimular la investigación científica en Colombia y qué hechos normativos y económicos han dado impulso al surgimiento de tales iniciativas. En segundo lugar, se explorarán las diferencias de opinión surgidas entre los investigadores que consideran necesario fomentar la creación de nuevas estrategias organizativas y quienes defienden la integridad de las existentes. Finalmente, se identificarán aquellos aspectos en los que la creación de formas organizacionales alternativas debería influir para ampliar la participación de los académicos en la investigación y mejorar la incidencia del conocimiento sobre los problemas sociales. Se argumentará que la importancia otorgada en la actualidad a la conformación de grupos colaborativos y multidisciplinares ha puesto en cuestión si las instituciones de investigación heterogéneas son más eficaces que aquellas especializadas y, por lo tanto, se hace necesario conocer la orientación de estos procesos de reestructuración organizativa en las universidades y su alcance en la materialización del carácter público del conocimiento.

\section{Metodología}

Para llevar a cabo este análisis se diseñó una aproximación cualitativa, basada en la realización de entrevistas semiestructuradas y en un ejercicio de observación etnográfica. Fueron entrevistados 30 investigadores vinculados a tres universidades colombianas, 
donde se han llevado a cabo recientemente procesos de reorganización de la actividad investigativa. Los participantes están adscritos como profesores, investigadores y estudiantes de doctorado a la Universidad de los Andes, a la Universidad Nacional de Colombia y a la Pontificia Universidad Bolivariana. Estas instituciones fueron seleccionadas para la realización del estudio porque lideran la producción de investigaciones en el ámbito académico colombiano y porque están ensayando nuevos modelos organizativos para fortalecer la investigación en las universidades. El grupo de individuos entrevistado estuvo conformado por 16 investigadores y 14 investigadoras, con niveles de experiencia investigativa que oscilaban entre los 30 y los tres años (tabla 1).

Después de revisar la literatura reciente y las reflexiones de los expertos sobre la gobernanza de la investigación universitaria, se diseñó un marco general de preguntas para explorar la visión de los participantes acerca de los cambios organizativos de su práctica y sus posibles consecuencias. Después de obtener respuestas sobre las primeras categorías de indagación se abrieron nuevas preguntas para profundizar en las perspectivas de los investigadores (tabla 2).
Se realizaron, además, diez ejercicios de observación etnográfica durante reuniones periódicas de los coordinadores de los CP de la Universidad Nacional (UNAL). Las observaciones permitieron recabar información general sobre cómo se delimitaron los CP en relación con otras unidades de investigación, qué áreas de la política pública deberían ser intervenidas, qué grado de interés despertaban entre los investigadores la conformación de los CP y qué actividades de investigación desarrollaban (tabla 3).

Durante las entrevistas y observaciones los participantes discutieron ideas, principios y políticas que se corresponden con maneras distintas de entender los problemas que se les plantearon y manifestaron opiniones opuestas con respecto a las demandas que debería satisfacer su práctica. Los investigadores relacionaron la obligación de cumplir metas contradictorias y la pérdida de incentivos para investigar, con la evolución de lógicas opuestas en el seno de las relaciones establecidas entre las universidades y sus académicos. Las consideraciones expresadas por los investigadores desvelan el surgimiento de tensiones dentro de las organizaciones académicas, tal como se mostrará a continuación.

Tabla 1. Características de los participantes

\begin{tabular}{|c|c|c|c|c|c|}
\hline Entrevistados & $\begin{array}{l}\text { Núm. de } \\
\text { participantes }\end{array}$ & Institución Universitaria & Ciudad & $\begin{array}{c}\text { Núm. de } \\
\text { participantes según } \\
\text { género (Masculino/ } \\
\text { femenino) }\end{array}$ & $\begin{array}{c}\text { Experiencia de } \\
\text { investigación (en } \\
\text { años) }\end{array}$ \\
\hline \multirow{3}{*}{$\begin{array}{l}\text { Profesor-investigador } \\
(\mathrm{PI})\end{array}$} & 10 & Universidad Nacional de Colombia & Bogotá & $7 \mathrm{M} / 3 \mathrm{~F}$ & Entre 10 y 30 \\
\hline & 5 & Universidad de los Andes & Bogotá & $1 \mathrm{M} / 4 \mathrm{~F}$ & Entre 10 y 20 \\
\hline & 2 & Pontificia Universidad Bolivariana & Medellín & $2 \mathrm{M}$ & Entre 7 y 16 \\
\hline \multirow[t]{3}{*}{ Investigador (IU) } & 3 & Universidad Nacional de Colombia & Bogotá & $2 \mathrm{M} / \mathrm{TF}$ & Entre 15 y 30 \\
\hline & 4 & Universidad de los Andes & Bogotá & $3 \mathrm{M} / \mathrm{TF}$ & Entre 10 y 15 \\
\hline & 1 & Pontificia Universidad Bolivariana & Medellín & $1 \mathrm{~F}$ & Entre 14 y 20 \\
\hline \multirow{2}{*}{$\begin{array}{l}\text { Estudiantes } \\
\text { de Doctorado } \\
\text {-investigador (ID) }\end{array}$} & 1 & Universidad Nacional de Colombia & Bogotá & $1 \mathrm{~F}$ & Entre 3 y 4 \\
\hline & 4 & Universidad de los Andes & Bogotá & $1 \mathrm{M} / 3 \mathrm{~F}$ & Entre 4 y 7 \\
\hline
\end{tabular}

Fuente: elaboración propia. 
Tabla 2. Marco general de preguntas

\begin{tabular}{|c|c|}
\hline Tema & Ejemplos de las preguntas \\
\hline Autonomía & $\begin{array}{l}\text { ¿Cree usted que las estructuras creadas recientemente por las universidades, tales como podrían ser los observatorios, centros de } \\
\text { pensamiento, centros de excelencia, entre otros, aseguran la autonomía de la investigación? Sí o no: ¿Por qué? ¿Cómo se manifiesta } \\
\text { concretamente dicho compromiso? } \\
\text { ¿Es posible decir que en la actualidad el mercado orienta de algún modo los objetivos de la investigación? Sí o no ¿Cómo? ¿Cuáles } \\
\text { serían las prioridades? ¿Cómo se establecen? }\end{array}$ \\
\hline Pertinencia & $\begin{array}{l}\text { ¿Cree usted que la investigación que se realiza en los Centros de Pensamiento podría lograr una mayor incidencia social que la que se } \\
\text { realiza en los espacios tradicionales de la academia? Sí o no ¿En qué medida? ¿Es necesario que la investigación universitaria tenga una } \\
\text { función pública? } \\
\text { ¿Piensa que en la actualidad los investigadores tienen la libertad para estudiar, evaluar críticamente y contribuir a la transformación } \\
\text { del país? }\end{array}$ \\
\hline Simplificación & $\begin{array}{l}\text { ¿La realización de investigaciones sobre problemas coyunturales podría ser de alguna manera incompatible con la rigurosidad de la } \\
\text { producción científica? Sí o no ¿De qué manera podría afectarla o contribuir a lograr sus objetivos? }\end{array}$ \\
\hline Experticia & $\begin{array}{l}\text { ¿Son los espacios de producción científica verticales y centralistas un obstáculo para la producción de conocimiento socialmente } \\
\text { pertinente? Sí o no ¿Qué ventajas o desventajas concretas tendría un modelo organizativo más pluralista? } \\
\text { ¿Qué consecuencias pueden tener los incentivos financieros dirigidos al desarrollo de investigaciones interdisciplinares sobre la } \\
\text { investigación disciplinar.. }\end{array}$ \\
\hline Comunicación & $\begin{array}{l}\text { ¿Cree que la transmisión oportuna de los resultados de las investigaciones tiene relación con la legitimidad que le otorgan diferentes } \\
\text { sectores sociales al conocimiento? } \\
\text { ¿Cree que las múltiples demandas que enfrentan los investigadores condicionan el alcance que los investigadores dan a sus estudios? } \\
\text { ¿Podría esta dinámica afectar la rigurosidad metodológica con la que se deberían realizar las investigaciones científicas? }\end{array}$ \\
\hline
\end{tabular}

Fuente: elaboración propia.

\section{Tabla 3. Resumen de los datos recolectados durante las observaciones de las actividades realizadas por los Centros de Pensamiento, UNAL}

\begin{tabular}{|l|l|}
\hline \multicolumn{1}{|c|}{ Categorías } & \multicolumn{1}{|c|}{ Información recolectada durante las observaciones } \\
\hline Conceptualización & Se recolectan datos sobre la conceptualización de los CP. \\
\hline Jerarquización de problemas & Se documentan las discusiones sobre agenda de investigación que debían seguir los CP. \\
\hline Compromiso de distintos estamentos & $\begin{array}{l}\text { Se intenta establecer si los CP concitan el interés activo de los investigadores y estamentos universitarios } \\
\text { heterogéneos. }\end{array}$ \\
\hline Actividades & Se identifican las actividades de investigación y divulgación desarrolladas por los CP. \\
\hline
\end{tabular}

Fuente: elaboración propia. 


\section{La gobernanza de la investigación universitaria hoy}

Los cambios organizativos que vienen ocurriendo en las universidades colombianas, los cuales se enfocan en el descentramiento de las bases institucionales mediante las cuales se había generado el conocimiento, podrían entenderse en parte como resultado de la dinámica global que anima el surgimiento de una nueva cultura epistémica para la producción científica. Ésta promueve la integración de los recursos cognoscitivos, de las alianzas interinstitucionales y de las capacidades investigativas universitarias en espacios heterogéneos, con el propósito principal de impulsar el crecimiento de la productividad académica y la articulación con los demás actores del sistema tecnocientífico (Katz y Martin, 1997: 8; Anderson, 2011).

En las universidades colombianas esta dinámica de transformación se viene expresando en la creación de espacios alternativos a aquellos en los que habitualmente se realizaba la investigación. Si de manera convencional las facultades e institutos se habían ocupado de la gobernanza y gestión de las actividades investigativas, ahora se promueve un reordenamiento de los recursos y el personal académico dedicado a esta actividad. Este reordenamiento busca fundamentalmente diversificar y flexibilizar la participación de los miembros de la comunidad universitaria en nuevos espacios de colaboración, tales como observatorios, centros de pensamiento, centros de excelencia y escuelas de pensamiento, entre otras estructuras organizativas.

A partir de esta orientación la Universidad Nacional de Colombia estableció en su Plan Global de Desarrollo 2014, que se crearían Centros de
Pensamiento con el propósito de posicionar la institución como principal entidad consultora del Estado. Para la formación de dichos centros la Universidad Nacional tomó como modelo los Think Tank, o un tipo de organizaciones que constituyen equipos multidisciplinares de científicos e intelectuales para elaborar análisis y recomendaciones solicitados por empresas privadas, laboratorios militares, instituciones gubernamentales y entidades consultoras (Abelson y Lindquist, 2000: 38). Entre 2014 y 2018, esta institución educativa promovió la formación de una Escuela de Pensamiento, con el fin de articular equipos de investigación multidisciplinares e integrar las actividades de veinticinco CP (UNAL, 2020a). La Universidad de los Andes cuenta, por su parte, con cuatro Centros de Pensamiento de larga trayectoria, y la Pontificia Universidad Bolivariana con dos instituciones de este mismo tipo. Estos centros reúnen en la actualidad numerosos grupos de trabajo, vinculados por el interés de avanzar en la formulación de recomendaciones para orientar la política pública (tabla 4).

Esta dinámica de reorganización viene siendo impulsada además por una nueva economía política de la producción de conocimiento, basada tanto en el aumento de los recursos de inversión para la investigación como en la formulación normativa que reglamenta el Sistema Nacional de Ciencia, Tecnología e Innovación (SNCTI). Dicha normatividad se ha enfocado principalmente en dos objetivos: por un lado, diversificar el tipo de actores que participan en la producción de conocimiento y, por otro, flexibilizar los canales a través de los cuales se organizan y articulan los actores del sistema. 
Tabla 4. Centros de Pensamiento de tres universidades colombianas, 2020

\begin{tabular}{|c|c|c|}
\hline Centros de Pensamiento unAL & $\begin{array}{c}\text { Centros de Pensamiento Universidad } \\
\text { de los Andes }\end{array}$ & $\begin{array}{c}\text { Centros de Pensamiento Pontificia } \\
\text { Universidad Bolivariana }\end{array}$ \\
\hline $\begin{array}{l}\text { CP Artes, patrimonio cultural y acuerdo social } \\
\text { CP Articulación en funciones misionales: Extensión } \\
\text { CP Articulación en funciones misionales: Investigación } \\
\text { CP Camilo Torres } \\
\text { CP Cátedra José Félix Patiño } \\
\text { CP Comunicación y ciudadanía } \\
\text { CP Derecho a la salud: sistemas y democracia } \\
\text { CP Desarrollo rural } \\
\text { CP Desarrollo sostenible } \\
\text { CP Educación } \\
\text { CP Ética de la investigación en modelos animales } \\
\text { CP Ética para la sostenibilidad ambiental } \\
\text { CP Evaluación y meritocracia } \\
\text { CP Gestión pública } \\
\text { CP Gran Caribe } \\
\text { CP Hábitat, ciudad y territorio } \\
\text { CP Integridad académica } \\
\text { CP Mares y océanos } \\
\text { CP Medicamentos } \\
\text { CP Nicanor Restrepo Santamaría } \\
\text { CP Política fiscal } \\
\text { CP Políticas públicas en educación superior } \\
\text { CP Propiedad intelectual } \\
\text { CP Responsabilidad y sostenibilidad de la industria minera } \\
\text { CP Seguimiento a los Diálogos de Paz }\end{array}$ & $\begin{array}{l}\text { - Centro de Estudios Estadounidenses (CEE) } \\
\text { - Congreso Visible ConPaz } \\
\text { - Centro de Construcción de Paz }\end{array}$ & $\begin{array}{l}\text { - Centro de Pensamiento Social } \\
\text { - Centro de Pensamiento Crítico }\end{array}$ \\
\hline
\end{tabular}

Fuente: elaboración propia.

A ritmo vertiginoso se han sucedido una serie de ajustes que buscan fortalecer el SNCTI y dotar con nuevos recursos al sector. En los últimos 10 años, la ley 1286 de 2009, procurando reforzar las disposiciones de la Ley 29 de 1990, trasformó a COLCIENCIAS en Departamento Administrativo, con el objetivo de que trabajara en la implementación de políticas que fortalecieran la incidencia del SNCTI en el entorno social y económico, y que generaran conocimientos para favorecer el crecimiento del sector productivo y la industria nacional. Así mismo, señaló en el inciso 2 del artículo 17 que el principal objetivo del SNCTI debía ser fomentar y consolidar centros, grupos de investigación y otras formas de organización que contribuyeran a fortalecer el sistema (Congreso Nacional de Colombia, 2009).

En este mismo periodo fueron aprobadas las leyes 633 de 2000 y 1819 de 2016, mediante las cuales se asignaron nuevos recursos financieros a la investigación, provenientes de fuentes alternativas a las que tradicionalmente otorgaban las entidades públicas y privadas. Con el otorgamiento de incentivos tributarios a quienes apoyaran el desarrollo de la ciencia y la tecnología, se creó una línea de recaudos que, aunque menos directa que las tradicionales, ha llegado a constituirse en pivote importante para la inversión en este campo (Congreso Nacional de Colombia, 2000, 2016). De otro lado, los recursos provenientes del Sistema General de Regalías han contribuido tanto a fortalecer la producción de conocimiento en las universidades como a generar capacidades de investigación en las áreas metropolitanas. Sólo entre 2017 y 2018 los departamentos invirtieron cerca de 70 billones de pesos en el desarrollo de infraestructura para investigación e innovación (COLCIENCIAS, 2018: 7). De igual manera, se informó que entre 2012 y 2020 se 
invirtieron 208 millones de dólares en proyectos para mejorar la calidad y el impacto de la investigación, se desarrollaron 33 planes departamentales en ciencia tecnología e investigación y se apoyó la realización de 1162 mesas de asistencia técnica en diferentes territorios a nivel nacional (COLCIENCIAS, 2020).

Aunque todavía es pronto para saber cuál ha sido el impacto de estas políticas, transcurridos apenas 10 años desde su promulgación, se puede decir que han impulsado algunos cambios en el modelo organizativo de la investigación. En la actualidad se busca la transición desde un sistema basado en el trabajo individual de los científicos, hacia un sistema basado en el trabajo de investigación colaborativo y multidisciplinar. Aunque en el pasado ya existieron algunas organizaciones de este tipo, los indicadores actuales muestran una tendencia sostenida en dirección a conformar espacios organizativos complejos, o grupos de investigación cada vez más heterogéneos en su composición y más provisionales en el tiempo (Room, 2011: 30). La evolución de esta dinámica ha sido tan patente que la multidisciplinariedad pasó de ser percibida como un instrumento útil para el fortalecimiento de la SNCTI, a ser considerada como fundamento para la generación de una nueva cultura organizativa en las instituciones universitarias.

\section{El debate sobre la reorganización de la investigación universitaria}

A medida que la colaboración y la multidisciplinariedad se han convertido en criterios centrales para apoyar el financiamiento de la investigación, han surgido críticas por parte de quienes consideran que esta orientación ha sido manipulada con el fin de facilitar la comercialización de la actividad investigativa.

La libertad académica es un principio amplio que protege el derecho de los profesores a investigar, publicar y enseñar material que puede ser impopular, controvertido o incluso incorrecto. Mientras la política es ampliamente aceptada, al menos en principio, las universidades deben lograr un equilibrio entre los derechos de los profesores a la libertad académica y las demandas de las partes interesadas internas y externas que intervienen en la producción científica (Entrevista a investigador).

Desde esta perspectiva la producción de conocimiento científico se estaría supeditando a intereses particularistas y los investigadores habrían abrazado una cultura epistémica que valora más los resultados comercializables que aquellos capaces de mover las fronteras del conocimiento (Sebastián, 2000: 140). Para algunos académicos, las experiencias de colaboración entre los investigadores no estarían conduciendo a una mayor diversificación del tejido científico, sino a establecer cooperaciones puntuales para cumplir con objetivos de corto plazo.

Las actuales políticas del conocimiento reconocen el valor potencial que podría tener la diversificación de las redes para aumentar la productividad científica, pero habría que hacer advertencias sobre sus posibles efectos. Por ejemplo, una política decía: 'Las redes diversas proporcionan métodos más rápidos y formas más directas de compartir información entre los investigadores', pero está orientación podría debilitar estructuras que tradicionalmente son favorecidas por organismos que dan prioridad a la financiación de redes especializadas y poco flexibles.

La historia de la institucionalización de las ciencias en América Latina demuestra la importancia que tuvieron las colaboraciones de los pares extranjeros en el diseño y consolidación de programas disciplinares, en la formación de estudiantes de doctorado, en el reconocimiento de incentivos a los científicos que apoyaran el desarrollo disciplinar a nivel local y en el desarrollo de proyectos editoriales especializados (Kleiche-Dray y Chiapa, 2016).

En todo caso, a medida que avanza la reestructuración multidisciplinar del sistema tecnocientífico y aumenta la demanda por conocimientos útiles, van surgiendo voces discordantes sobre la puesta en 
marcha de estos procesos de reconfiguración institucional. Si se tiene en cuenta que la diversificación de las colaboraciones y el aumento de su complejidad parecen obligatorias, debido entre otras causas a la creciente interdisciplinariedad de la experimentación y a la sofisticación de las bases tecnológicas de la investigación, es indispensable identificar los argumentos que forman parte del debate entre los investigadores (Brewer, 1999: 328). En este trabajo se han identificado concretamente tres tensiones principales.

\section{Tensión entre uniformidad y pluralidad de los grupos científicos}

Se sabe que en el pasado las universidades hicieron esfuerzos significativos para estructurar cuerpos académicos uniformes y especializados, mientras que en la actualidad procuran organizar centros de pensamiento que integren pares plurales y desarrollen agendas dirigidas a públicos heterogéneos. Sin embargo, a la mayoría de los investigadores entrevistados les preocupa que la extensión de la colaboración entre científicos heterogéneos pudiera dar como resultado versiones simplificadas del conocimiento. Su visión parece implicar que el lugar natural para desarrollar la investigación serían los institutos especializados, donde los académicos comparten altos niveles de formación y los mismos criterios de validación del conocimiento. En estos espacios, los científicos tendrían mayores ventajas para lograr objetivos comunes y para elaborar productos más complejos y especializados. Dicho en otras palabras, se piensa que los grupos heterogéneos no comparten criterios idénticos para validar la producción científica y que ello podría tener efectos negativos sobre la calidad de los resultados.

Esta percepción fue puesta en cuestión por otros investigadores mediante dos argumentos distintos: en primer lugar, advirtiendo que la extensión de los criterios de validación tiene relación con el alcance de la investigación y, segundo, recordando que en la actualidad se ha hecho cada vez más difícil defender la existencia de parámetros asépticos y aproblemáticos para medir el supuesto carácter estable y consistente del conocimiento. Se sugiere, por ejemplo, que mientras la evaluación de la producción científica orientada a hacer aportes teóricos y metodológicos requeriría la evaluación experta de los pares disciplinares, la evaluación de racionalizaciones sobre aspectos convencionales del conocimiento podría no sólo ser arbitrada por grupos de académicos plurales, sino enriquecida con la colaboración de pares interinstitucionales. La pregunta planteada por investigadores adscritos a los CP es por qué habría que ignorar el hecho de que versiones simplificadas del conocimiento pueden ser útiles para gestionar proyectos colaborativos entre audiencias diversas (Nugroho et al., 2018: 42).

\section{Tensión entre experticia y practicidad}

Una segunda tensión es discutida por quienes consideran que hay una orientación ideológica en la tendencia a privilegiar un tipo de organización que favorezca la investigación coyuntural, en detrimento de la que tradicionalmente sustentó el desarrollo del conocimiento científico. Algunos de los investigadores temen que el trabajo de los académicos interesados en producir investigaciones teóricas, desde enfoques disciplinares o multidisciplinares, no sea igualmente valorado en un contexto en el que se priorice la resolución de problemas puntuales o la producción de resultados prácticos. Casi la mitad de los entrevistados argumentó que el paso de una investigación empírica a otra, de manera desordenada, tratando de resolver problemas de la esfera política, podría propiciar la relajación de los principios teóricos que deben guiar la investigación. Es probable que este prejuicio tenga su origen en la convicción proverbial de que debe existir una separación tajante entre la política y el trabajo científico, pero los investigadores continúan siendo fieles a la idea de que se deben producir modelos sofisticados y elegantes para resolver los problemas del conocimiento 
y abstenerse de establecer conclusiones sólidas con base en escasos datos empíricos (López, 2016).

Algunos investigadores observan que, si sus colegas se abrieran con más frecuencia a los cuestionamientos de la política, quizá podrían reconocer la poca sostenibilidad que tienen algunos de los trabajos teóricos fuera del ejercicio académico. En su opinión, muchos de los que defienden la preeminencia de los trabajos teóricos, por encima de otro tipo de estudios, en realidad se limitan a la construcción de grandes simplificaciones, escritas con el apoyo de largas bibliografías y sin ninguna contrastación material. Proponen, incluso, que el uso de metodologías comúnmente aplicadas en la investigación política quizá podría ayudar a identificar problemas que no han sido vistos en las densas teorizaciones de los expertos. Para autores como Christopher Pollitt (2020), cuanto más evidente se hace el carácter ubicuo del conocimiento en la actualidad, mejor se entiende la importancia de conocimientos prácticos que por generaciones han contribuido a la sostenibilidad planetaria. Las comunidades académicas habrían sistematizado aparatos analíticos casi incuestionables, pero se considera que algunos son demasiado generales para atender problemas puntuales, y que la integración de las competencias relacionadas con el saber hacer es uno de los retos más importantes que tienen las instituciones de educación superior.

\section{Tensión entre comunicación e investigación}

La necesidad de comunicar los resultados de la investigación a audiencias amplias también ha generado controversias, pues se les crítica a los CP haber eliminado la separación tradicional entre la divulgación de los resultados y el proceso de investigación. Se afirma que en estos espacios se ha subordinado la rigurosidad académica a las demandas puntuales de las editoriales y que en consecuencia se ha roto la necesaria separación entre la comunicación de los resultados y el proceso de investigación. Dicha separación se habría mantenido como una característica esencial de la ciencia normal, porque la divulgación atomizada de resultados no contribuiría a dinamizar el proceso acumulativo del conocimiento, o al menos así lo habrían entendido las universidades cuando se esforzaron por estructurar y gestionar procesos de divulgación para investigaciones de mediano y largo plazo.

Una mirada a las actividades divulgativas realizadas por los CP de la Universidad Nacional muestra, sin embargo, que éstos utilizan los mismos medios de comunicación que el resto de la comunidad académica. Pese a los requerimientos para aumentar el volumen de información y mejorar su oportunidad, los sistemas de divulgación continúan siendo convencionales y manteniendo los mismos plazos de publicación. A corte de 2019 los CP reportaron la elaboración de 13 documentos de política y cuatro libros, presencia en 88 foros y congresos, presencia en un medio de comunicación, la realización de 34 productos digitales y la programación de 362 eventos de discusión, la mayoría de ellos conferencias, encuentros y foros estratégicos (Universidad Nacional de Colombia, 2019: 37).

Los investigadores de los CP manifestaron, además, que para responder a las críticas han tratado de definir las estrategias de comunicación desde el inicio de la investigación, teniendo cuidado de distinguir entre aquellas que se utilizarán para dar a conocer los resultados de trabajos coyunturales y aquellas que divulgarán los hallazgos de las investigaciones de larga duración. Si se ha decidido dar prioridad a la realización de asesorías, a la ejecución de interventorías o a la elaboración de proyectos financiados con recursos de regalías, se diseña desde el inicio del proyecto una estrategia de comunicación dirigida a difundir resultados en el corto plazo y entre audiencias amplias. Además, para responder de manera oportuna a las demandas de información coyuntural también han recurrido a la reedición de textos que todavía discuten problemas actuales. 
La actividad de divulgación más popular en los CP ha sido la organización de congresos, porque a juicio de los investigadores son más efectivos para discutir el conocimiento de manera casi inmediata y para mantener conversaciones con actores involucrados en la gestión de la política pública. Desde el punto de vista de los críticos, la preferencia por esta estrategia comunicativa no favorece la continuación de conversaciones e intercambios en el mediano plazo, y tampoco fomenta la concertación de nuevos proyectos. Para dar a conocer portafolios de servicios y gestionar alianzas con diferentes actores del SNCTI, en apoyo de las labores realizadas por los CP, las universidades han intentado inclusive contratar empresas especializadas en la organización de mesas de trabajo con administradores públicos, planeadores, autoridades municipales y asociaciones civiles (Montoya, 2019).

De este modo, es claro que las iniciativas institucionales para avanzar en la generación de alianzas móviles y flexibles, experimentando con colaboraciones menos duraderas, con la investigación de temáticas más diversas y con la integración de equipos más provisionales, no han recibido la aprobación unánime de la comunidad académica. Coinciden, eso sí, en que la creación de nuevos espacios no debería servir únicamente para reunir una mayor cantidad de especialistas, sino para dinamizar los recursos cognoscitivos de los investigadores. Como ha señalado Bruno Latour (1987: 76), recursos tales como bibliografías, máquinas, laboratorios, centros de estudio, y medios de divulgación son movilizados por los miembros de diferentes grupos académicos, haciendo que la conformación de espacios colaborativos y multidisciplinares ciertamente beneficie el desarrollo tecnocientífico.

\section{Puntos críticos sobre los que debería incidir la reorganización}

Las reflexiones de los investigadores permiten identificar tres aspectos críticos sobre los que deberían influir las futuras estrategias organizativas de las universidades: el fomento de la libertad de asociación entre los científicos, la independencia para determinar el espacio de circulación de sus productos e intermediarios y la superación del control monopólico que las disciplinas han ejercido sobre la investigación. Es probable que la preocupación por aumentar la productividad científica haya conducido a las instituciones por caminos estrechos, impidiendo estimar la importancia de la circulación y el posicionamiento del conocimiento. Es cierto, por ejemplo, que se han realizado enormes y complicadas misiones para definir cuáles deben ser las agendas de investigación, descuidando el hecho de que el campo científico va acotando los intereses hasta definir lo que es legítimo y valioso investigar (Bourdieu, 1997: 12).

Un análisis de las agendas investigativas priorizadas por los actores del SNCTI, las agencias de cooperación y algunas universidades latinoamericanas, muestra coincidencias generales en las áreas de investigación en las que se enfocan. La salud, el desarrollo económico sustentable, las ciencias agrarias, el medio ambiente y la energía se constituyen en focos de una agenda orientada a resolver problemas con un nivel de incidencia global (De Haas, 2017: 53-54). La biotecnología y la transformación informática se identifican como insumos necesarios para la innovación empresarial e industrial, y la investigación sobre los recursos hídricos, naturales y minerales se define como un recurso crucial para el crecimiento económico y la mitigación de la pobreza. La contribución de las artes a la creación de nuevas industrias y emprendimientos se vislumbra como un sector en crecimiento y se espera que las ciencias sociales tengan mayor impacto en la gestión pública y en el fortalecimiento institucional (tabla 5).

Discusiones como las que ha promovido la llamada "Misión de Sabios" de 2019, si bien no carecen completamente de importancia, tampoco aportan demasiado al diseño de estrategias concretas para dinamizar la organización de la investigación. Habría 
que preguntarse cómo participar en la realización de tales agendas, teniendo en cuenta que las relaciones entre los científicos ocurren en condiciones asimétricas. Generalmente se han diseñado planes para incrementar el volumen de resultados científicos, cuando en la actualidad es más urgente transformar las condiciones de su producción. La densificación de las interacciones entre los investigadores podría ser un principio de acción necesario para aumentar los recursos materiales y cognoscitivos dedicados a la investigación y para generar conocimientos más diversos (Chompalov y Shrum, 1999: 339).

Tabla 5. Comparación de la agenda investigativa colombiana con la de algunas universidades latinoamericanas

\begin{tabular}{|c|c|c|c|c|c|}
\hline \multicolumn{6}{|c|}{ Agenda de investigación de los actores del sNCTI - Colombia } \\
\hline $\begin{array}{c}\text { Misión de sabios } \\
2019\end{array}$ & $\begin{array}{c}\text { Agenda SNCT según } \\
\qquad Z\end{array}$ & $\begin{array}{l}\text { oCDE Objetivos de } \\
\text { desarrollo sostenible }\end{array}$ & Colciencias Ley 1286 & $\begin{array}{l}\text { Agendas de } \\
\text { Conocimiento - } \\
\text { UNAL 2002-2014 }\end{array}$ & $\begin{array}{c}\text { Centros de } \\
\text { Pensamiento unAL } \\
\text { 2014-presente }\end{array}$ \\
\hline $\begin{array}{l}\text { Ciencias Sociales y } \\
\text { Desarrollo Humano } \\
\text { con Equidad }\end{array}$ & $\begin{array}{l}\text { Estado simple: } \\
\text { carga regulatoria del } \\
\text { gobierno }\end{array}$ & $\begin{array}{c}\text { Paz, justicia e } \\
\text { instituciones sólidas }\end{array}$ & $\begin{array}{c}\text { Ciencias sociales y } \\
\text { humanas }\end{array}$ & $\begin{array}{l}\text { Estado: sistemas } \\
\text { políticos y Jurídicos }\end{array}$ & Gestión pública \\
\hline $\begin{array}{c}\text { Ciencias de la Vida } \\
\text { y Salud }\end{array}$ & $\begin{array}{l}\text { Transporte e } \\
\text { integración }\end{array}$ & Salud y bienestar & $\begin{array}{c}\text { Ciencia y tecnología } \\
\text { en salud }\end{array}$ & Salud y vida & $\begin{array}{l}\text { Derecho a la salud, } \\
\text { sistemas y democracia }\end{array}$ \\
\hline $\begin{array}{c}\text { Océanos y Recursos } \\
\text { Hídricos }\end{array}$ & Transformación digital & $\begin{array}{l}\text { Agua limpia y } \\
\text { saneamiento }\end{array}$ & $\begin{array}{c}\text { Desarrollo } \\
\text { tecnológico, industria } \\
\text { y calidad }\end{array}$ & $\begin{array}{l}\text { Tecnologías de } \\
\text { la información y } \\
\text { comunicaciones }\end{array}$ & $\begin{array}{l}\text { Comunicación y } \\
\text { ciudadanía }\end{array}$ \\
\hline $\begin{array}{c}\text { Ciencias Básicas y } \\
\text { Espacio }\end{array}$ & Educación & Educación de calidad & $\begin{array}{c}\text { Estudios científicos de } \\
\text { la educación }\end{array}$ & Energía & Educación \\
\hline Energía Sostenible & $\begin{array}{c}\text { Mercados e inversión } \\
\text { extranjera }\end{array}$ & Vida submarina & Biotecnología & Biotecnología & Mares y océanos \\
\hline $\begin{array}{c}\text { Bioeconomía y Medio } \\
\text { ambiente }\end{array}$ & Desarrollo sostenible & Acción por el clima & $\begin{array}{c}\text { Ciencia y tecnología } \\
\text { agropecuaria }\end{array}$ & $\begin{array}{c}\text { Ciencias agrarias y } \\
\text { desarrollo rural }\end{array}$ & Desarrollo rural \\
\hline $\begin{array}{l}\text { Tecnologías } \\
\text { convergentes e } \\
\text { industrias }\end{array}$ & $\begin{array}{l}\text { Desarrollo } \\
\text { tecnológico e } \\
\text { innovación }\end{array}$ & $\begin{array}{l}\text { Ciudades y } \\
\text { Comunidades } \\
\text { sostenibles }\end{array}$ & $\begin{array}{l}\text { Fomento de la } \\
\text { formación en } \\
\text { investigación }\end{array}$ & $\begin{array}{l}\text { Desarrollo } \\
\text { organizacional } \\
\text { económico e } \\
\text { industrial }\end{array}$ & Desarrollo sostenible \\
\hline \multirow[t]{4}{*}{$\begin{array}{c}\text { Industrias creativas y } \\
\text { culturales }\end{array}$} & Economía naranja & $\begin{array}{c}\text { Producción y } \\
\text { consumo responsable }\end{array}$ & $\begin{array}{c}\text { Ciencia y tecnología } \\
\text { del mar }\end{array}$ & $\begin{array}{c}\text { Ciencia y tecnología } \\
\text { de materiales y } \\
\text { minerales }\end{array}$ & Medicamentos \\
\hline & $\begin{array}{l}\text { Transformación } \\
\text { empresarial y Pymes }\end{array}$ & Industria e innovación & Ciencias Básicas & Artes y cultura & $\begin{array}{c}\text { Artes, patrimonio, } \\
\text { cultura y acuerdo } \\
\text { social }\end{array}$ \\
\hline & Desarrollo agrícola & $\begin{array}{l}\text { Energía asequible y no } \\
\text { contaminante }\end{array}$ & $\begin{array}{l}\text { Investigación en } \\
\text { Energía y minería }\end{array}$ & $\begin{array}{l}\text { Hábitat, ciudad y } \\
\text { territorio }\end{array}$ & $\begin{array}{l}\text { Hábitat, ciudad y } \\
\text { territorio }\end{array}$ \\
\hline & & $\begin{array}{l}\text { Vida de ecosistemas } \\
\text { terrestres }\end{array}$ & & $\begin{array}{l}\text { Ambiente y } \\
\text { biodiversidad }\end{array}$ & $\begin{array}{l}\text { Sostenibilidad } \\
\text { ambiental }\end{array}$ \\
\hline
\end{tabular}


Tabla 5. Comparación de la agenda investigativa colombiana con la de algunas universidades latinoamericanas (continuación)

\begin{tabular}{|c|c|c|c|}
\hline \multicolumn{4}{|c|}{ Agendas de otras universidades de América Latina } \\
\hline UBA-CONICET & Universidad de Chile & CONACYT - MéXico & Universidad de Sao Paulo \\
\hline Sociales y humanidades & $\begin{array}{c}\text { Divulgación y valoración de ciencia } \\
\text { y tecnología }\end{array}$ & Materiales avanzados & Biocombustibles \\
\hline Ciencias agrarias & Desarrollo tecnológico & $\begin{array}{l}\text { Tecnología de la información y } \\
\text { comunicaciones }\end{array}$ & $\begin{array}{c}\text { Tecnología de la información y la } \\
\text { comunicación }\end{array}$ \\
\hline Ingeniería y materiales & Investigación y desarrollo en salud & Biotecnología & Energías renovables \\
\hline Ciencias biológicas y de la salud & $\begin{array}{l}\text { Formación de capital humano } \\
\text { avanzado }\end{array}$ & Diseño y procesos de manufactura & Ciencias agrícolas \\
\hline Ciencias exactas y naturales & & Infraestructura & Biotecnología y nanotecnología \\
\hline & & Desarrollo rural & Ciencias de la salud \\
\hline & & & Biodiversidad y recursos naturales \\
\hline & & & Meteorología \\
\hline & & & Programa espacial \\
\hline
\end{tabular}

Fuente: elaboración propia.

\section{La libertad para asociarse con otros actores}

La transformación organizativa de la investigación universitaria podría orientarse a constituir equipos de trabajo complejos y, por consiguiente, a desarrollar nuevas habilidades y destrezas prácticas. Aunque a nivel nacional ha habido algún interés por diseñar estrategias para fortalecer las capacidades de los actores del SNCTI, las políticas de investigación se han enfocado básicamente en evaluar y fortalecer los aspectos relacionados con la productividad científica. Los proyectos para apoyar la creación de incubadoras empresariales, centros de excelencia y centros de pensamiento, entre otras estrategias para dinamizar el sistema, apenas comienzan a mostrar su potencial integrador, si bien es necesario advertir que benefician particularmente a aquellas instituciones con mejores capacidades instaladas.

La última evaluación de resultados que se realizó a los proyectos financiados con recursos del Sistema Nacional de Regalías señala que las capacidades departamentales de innovación social continúan siendo dispares, y que no ha habido variaciones significativas 
en el Índice de Desarrollo para la Innovación, pese a la inversión que han realizado los departamentos. Sólo Bogotá y Antioquia registran un alto nivel de crecimiento de este indicador, muy por encima de la media departamental. Además, el mismo informe señala que sólo el $12 \%$ de los proyectos ejecutados generó impacto regional, habiéndose medido la incidencia geográfica y el uso real de los resultados de los proyectos. Las metas proyectadas para la realización de avances en investigación e innovación social a nivel municipal se cumplieron escasamente en un $20 \%$, y los proyectos ejecutados generaron articulación entre actores del SNCTI sólo en un 64\% de los casos (García, 2018: 21).

Estos indicadores muestran un panorama negativo de la participación regional en actividades tecnocientíficas, sumado al hecho de que normalmente los actores locales no tienen injerencia en la formulación de los proyectos. Aunque son las entidades territoriales las encargadas de ejecutarlos, los actores locales sólo son consultados cuando los proyectos ya han sido aprobados. Esto no sólo dificulta que los resultados lleguen a convertirse en un aporte al cumplimiento de las metas sectoriales en las regiones, sino que limita las posibilidades de articular el conocimiento local al SNCTI. La disparidad en la asignación de los recursos financieros para la investigación también ha contribuido a crear nodos de poder diferenciales, por lo que es aún más urgente formular políticas que contribuyan a compensar las diferencias y, particularmente, a fomentar la colaboración entre equipos de investigación heterogéneos (Suarez y Dutrénit, 2014: 20).

El fortalecimiento de la investigación universitaria a nivel regional podría estar apoyada en una política de integración, que asigne recursos para estimular la participación en grupos y que haga de la libertad de asociación el centro de una estrategia para realizar el potencial de los científicos locales. La creación de espacios colaborativos transregionales y multidisciplinares podría equilibrar el escaso desarrollo científico en las localidades, porque estimularía el desarrollo de compatibilidad técnica y la movilización de los sectores interesados en financiar la generación de conocimiento. A partir de allí se podrían gestionar alianzas, contrarrestar debilidades y mostrar las habilidades de los investigadores. Las universidades, como actores diferenciales del SNCTI, enfrentan el reto de animar la creación de espacios multiinstitucionales, y facilitar el tránsito del conocimiento local tanto al sistema científico nacional como global. El resultado final podría ser doble, por un lado, se reproducirían nuevas alianzas y articulaciones entre los académicos y, por otro, se reducirían las debilidades de la cultura técnica local para adaptarla a la práctica investigativa.

\section{Libertad para la circulación de productos e intermediarios}

El fomento de espacios colaborativos y multidisciplinares también podría estimular la coproducción de conocimientos que sirvan como insumos para iniciar nuevos análisis o para apoyar la realización de los que ya están en marcha. Las interacciones entre los investigadores y sus pares se limitan con frecuencia a la comunicación conjunta de sus trabajos académicos. Sin embargo, desde una perspectiva integradora habría que hacer énfasis en la necesidad de desarrollar procesos abiertos a la coproducción de conocimiento y a la realización de estudios que apoyen las agendas de investigación locales. El diseño conjunto de estudios podría no sólo atravesar las barreras disciplinares, sino ayudar a superar los escollos geográficos y a incluir a los investigadores aislados (Isoglio y Echaverry-Mejía, 2019: 32-33).

El fortalecimiento de estrategias para hacer uso compartido de laboratorios certificados y realizar pruebas de estandarización podría generar crecientes interacciones entre los investigadores. Habría que evaluar los criterios de reciprocidad mediante los cuales se garantizaría el equilibrio en estas relaciones de intercambio, pero no cabe duda de que ubicarse en la órbita de diversas redes de laboratorios podría crear 
nuevos canales de circulación para la producción científica. Bruno Latour describe esta dinámica como la creación de "un breve circuito, establecido entre una gran cantidad de grupos" (1983: 9). La acción al interior de los laboratorios se orientaría por el interés de los investigadores en enrolar a colegas destacados e impulsarlos a investigar lo que ellos consideren relevante. El ensamblaje práctico de investigadores e instrumentos provenientes de diferentes laboratorios sería crucial para incrementar la producción de conocimiento, porque reuniría y activaría las habilidades y destrezas de grupos de trabajo diversos.

En la actualidad se hacen ensayos pioneros para establecer algunas colaboraciones técnicas entre las universidades y las empresas. La calibración de equipos de control numérico, el mantenimiento de instrumentación, la estandarización de pruebas, las mediciones de rendimiento y la evaluación de materiales, entre otros procesos, son algunas de las prácticas en las que más se evidencia colaboración entre los laboratorios universitarios y la industria. También han surgido algunas fundaciones que se encargan de gestionar intercambios entre universidades e institutos de investigación internacionales, buscando extender las redes de circulación del conocimiento local. Aunque todavía no convergen en estas asociaciones todos los agentes que deberían movilizarse, algunas alianzas se han mantenido en el mediano plazo.

Han aparecido proyectos de publicación que involucran a profesores, estudiantes, investigadores, comunicadores y técnicos. Desafortunadamente, los esfuerzos de las revistas por controlar a los investigadores a través de los índices de impacto marchan en contravía de estas dinámicas de colaboración. Aquellas publicaciones mejor situadas en los rankings nacionales e internacionales monopolizan la publicación de artículos y los índices de citación. Estar informado de lo que se lee, y a quienes se lee, asegura que las propias publicaciones de los lectores tengan mayores posibilidades de ser evaluadas y recibidas de manera positiva por la comunidad académica.
Las cuotas de rechazo y la espera para que los artículos sean publicados aumenta, y la aceptación de las contribuciones se convierte en un privilegio (Nelson, 2009: 67). Además, las universidades carecen de mecanismos efectivos para animar la comunicación entre los investigadores y los gestores políticos, en un contexto en el que se exige contribuir con el acceso libre al conocimiento abierto y, al mismo tiempo, se respeta el monopolio de la divulgación científica.

Al implementar metodologías de "diálogos regionales", los CP han intentado propiciar encuentros con planeadores municipales y autoridades locales, con el fin de identificar intereses convergentes con la agenda pública y mejorar las capacidades de la universidad para responder a sus demandas. Actuando a través de unidades especializadas como los Centros de Liderazgo y Gestión, cuyo trabajo consiste en facilitar diálogos entre diferentes actores para trabajar cooperativamente en la gestión de retos complejos, las universidades han tratado de dar a conocer sus portafolios de servicios y armonizarlos con las necesidades locales. En la práctica, las relaciones que han surgido en el marco de estos encuentros se han ido haciendo más afirmativas y han surgido algunas experiencias de cooperación para el diseño de proyectos a financiar con recursos del Sistema General de Regalías.

Algunos investigadores piensan que planear encuentros regionales entre las universidades y las autoridades locales podría ayudar a cerrar brechas entre los diferentes actores del sistema y a desarrollar las capacidades de las entidades educativas para gestionar objetivos de alto impacto. Aunque los intermediarios se comprometen a dinamizar la comunicación y el intercambio de información, su intervención tampoco ha estado exenta de polémicas, ya que no todos les consideran imparciales frente a otros actores del sistema (Newman et al., 2012: 19). Por otro lado, en la medida en que los intermediarios promueven básicamente la conformación de escenarios para compartir conocimientos y experiencias, se señala que no es fácil evaluar la efectividad de esta gestión puntual 
o la evolución de los procesos iniciados (Torres, 2016: 289). Aun con estas reticencias, la negativa a involucrar intermediarios en la gestión del conocimiento podría marchar en contravía de la democratización de los circuitos para la comunicación científica.

\section{Superación del control monopólico de la investigación disciplinar}

Aunque la comunidad universitaria había estado tradicionalmente orientada a desarrollar las disciplinas, durante las últimas décadas ha mostrado una mayor disposición para discutir cómo generar proyectos multidisciplinares. Sin embargo, no parece que este debate se haya traducido en acciones concretas para tornar definitivamente hacia este propósito. En términos generales, las instituciones universitarias no han creado condiciones substancialmente diferentes para transformar las estrategias organizativas que permitieron desarrollar las disciplinas. Las universidades siguen teniendo dificultades para vincular nuevos estudiantes de posgrado a procesos de formación e investigación interdisciplinar, al mismo tiempo que persisten en la conformación de departamentos poco diversos y no consiguen armonizar las capacidades desarrolladas con la dinámica organizativa del campo científico globalizado (Vessuri, 2013: 204).

El tamaño reducido de los departamentos y la poca diversidad en los perfiles de sus profesores, los cuales rara vez suman un grupo con más de 20 personas, pueden ser obstáculos no sólo para la diversificación del conocimiento, sino para el diálogo de saberes y para la jerarquización de funciones académicas. En la Universidad Nacional el número de profesores de planta por departamento es en promedio de 28, mientras que en la Universidad de los Andes es de 29, ambas por encima del promedio nacional (Universidad Nacional de Colombia, 2020b; Universidad de los Andes, 2020). Algunos países, como Alemania, muestran una separación creciente del trabajo formativo e investigativo, dedicando casi un $40 \%$ de los recursos de inversión a institutos fuera de las universidades, mientras que otros países, como Canadá y Estados Unidos, han sacado amplias ventajas de integrar la formación y la investigación (Becker y Bauer, 2018: 353). Alemania se ha mantenido reticente a la integración de las funciones universitarias, con el argumento de que este proceso devalúa la formación y parcializa los resultados de la investigación al circunscribirla a unas pocas áreas atractivas (Vessuri, 2014: 172).

Idéntica razón mencionaron los investigadores entrevistados al defender su postura contra la integración de las actividades universitarias. Las universidades fuertemente jerarquizadas en el ámbito interno, es decir, donde hay marcados rangos entre departamentos y facultades, dificultan la integración interdisciplinar y desaprovechan su fuerza creativa. Los institutos especializados han ofrecido resistencia a la búsqueda de interlocutores fuera de las áreas disciplinares que ya robustecieron y los departamentos tampoco se han diversificado de manera decidida. Estas jerarquizaciones se han utilizado comúnmente para asegurar el monopolio de las recompensas provistas por la ciencia normal, pero se pasa por alto el hecho de que la carrera frenética para acumular puntos ha distraído a los investigadores de su trabajo para conseguir la prioridad científica.

Siendo la universidad un lugar diferencial para realizar la investigación, por cuanto suma una gran diversidad disciplinar y posee una gran fuerza imaginativa, podría tomar el riesgo de desarrollar esa capacidad. Una universidad que intente integrar su potencial interdisciplinar podría incluso superar la procedencia epistemológica del saber. La incorporación académica de los saberes tácitos o de los conocimientos tradicionales, los cuales han hecho importantes aportes al conocimiento socioecológico, al desarrollo sustentable y a la farmacopea mundial, podría dar lugar a un proceso de aprendizaje colaborativo que trascienda las diferencias epistémicas y se ubique en la perspectiva de las comunidades locales. La diversidad del conocimiento tradicional es un 
factor que podría promoverse como estrategia para evitar la uniformidad y limitar el monopolio de la investigación disciplinar. Además, su riqueza y diversidad cultural podría ser justamente el activo con el que la ciencia haga frente al carácter simplificador de las demandas del mercado.

Los monopolios institucionales son obstáculos difíciles de superar porque han sido erigidos a lo largo de varias generaciones. De hecho, puede que la creación de capacidades para la participación, la deliberación y el trabajo colaborativo, por parte de quienes deben concebir la política de investigación, sea apenas un primer paso en la evolución de la ciencia abierta (Smith, 2017: 67-68). Por otro lado, tampoco será suficiente redireccionar la producción científica hacia la satisfacción de intereses sociales, si no crecen y se redistribuyen las capacidades para producir conocimiento. Más que entusiastas exposiciones de propósitos sobre la necesidad de estimular la integración de los científicos, se requeriría desarrollar estrategias concretas para transformar las condiciones que dieron lugar a jerarquizaciones y garantizar igualdad de oportunidades a quienes desean constituir formas de asociación multidisciplinares.

\section{Consideraciones finales}

La subordinación de la investigación a la autoridad de los departamentos y facultades universitarias quizá ha concedido a los investigadores poca autonomía para avanzar en la praxis gerencial de su conocimiento y en la estimación de las consecuencias que tendría la flexibilización de las normas organizativas. Sin embargo, la transformación de la estructura institucional para crear espacios de investigación más flexibles tampoco ha recibido completa aprobación por parte de algunos investigadores, quienes la consideran una estrategia fútil para responder a las exigencias del mercado y de los gobiernos. La mayoría está de acuerdo en que, si se supeditara el apoyo de la investigación a demandas particulares o intereses económicos, se afectaría gravemente la autonomía universitaria y se obstaculizaría la posibilidad de elaborar conocimiento científico de frontera.

Los debates han expuesto las divergencias existentes dentro de la comunidad académica sobre los caminos más adecuados para democratizar el ejercicio de la actividad investigativa. La libertad para asociarse con pares de diferentes instituciones, la autonomía para elegir los circuitos de comunicación y los intermediarios de sus proyectos, y las iniciativas para superar el monopolio disciplinar se dejan ver como necesidades palpables para materializar el trabajo colaborativo y multidisciplinar. Flexibilizar la organización de la investigación tendría que ser considerado como una prioridad para las universidades, si ello contribuyera a la multiplicación de conexiones, a la acumulación de experiencias, a la incorporación de los saberes locales y, en general, a la ampliación de las capacidades y habilidades del tejido tecnocientífico.

De este modo, es importante comprender lo que está en juego para quienes discrepan de las orientaciones institucionales, porque cada vez que la balanza se inclina lo suficiente de un lado o del otro, se vuelve más difícil para las universidades responder de manera apropiada a las críticas y exigencias de los reclamantes. Lo cierto es que buscar acuerdos para generar combinaciones entre diferentes equipos de trabajo podría facilitar el desarrollo de agendas conjuntamente priorizadas por la comunidad académica. Fomentar espacios en los que converjan los intereses de los académicos, de los planificadores y de otros actores políticos podría ser lo que convierta el conocimiento en un bien público. El carácter público de la investigación y de sus resultados ciertamente no debería definirse por el origen oficial de los fondos que se le invierten, sino por la capacidad de las instituciones para hacer que el conocimiento tenga incidencia sobre la realidad social y contribuya a trasformar las condiciones de vida de los ciudadanos. 


\section{Referencias}

Abelson, Donald y Evert Lindquist (2000), "Think tanks in North America", en R. Weaver y J. Gann (eds), Think tanks and civil societies: catalysts for ideas and action, Londres/ Nueva York, Routledge, pp. 37-66.

Anderson, Winston et al. (2011), "Changing the culture of science education at research universities", Science, vol. 331, núm. 6014, pp. 152-153.

Becker, Stefan y Michael Bauer (2018), “¿Public administration in Germany: precarious present, promising future?", en Geert Bouckaert and Werner Jann (eds.), European perspectives for public administration, Lovaina, Leuven University Press, pp. 351-365.

Bourdieu, Pierre (1997), Los usos sociales de la ciencia, Buenos Aires, Ediciones Nueva Visión.

Brewer, Garry D. (1999), "The challenges of interdisciplinarity", Policy Sciences, núm. 32, pp. 327-337.

COLCIENCIAS (2018), Informe de gestión y resultados 2017 -

2018, Bogotá, Departamento Administrativo de Ciencia Tecnología a Innovación, Universidad Nacional de Colombia.

COLCIENCIAS (2000), COLCIENCIAS en cifras, Bogotá, Ministerio de ciencia y Tecnología, <https://minciencias. gov.co/la-ciencia-en-cifras/presupuesto-colciencias> [Consulta: octubre de 2020].

Congreso Nacional de Colombia (2016), Ley 1819 de 2016, Bogotá, Congreso Nacional de Colombia, <http://www.secretariasenado.gov.co/senado/basedoc/ ley_1819_2016.html> [Consulta: enero de 2020].

Congreso Nacional de Colombia (2009), Ley 29 de 2009 por la cual se modifica la Ley 29 de 1990 que transforma a Colciencias en Departamento Administrativo, se fortalece el Sistema Nacional de Ciencia, Tecnología e Innovación en Colombia y se dictan otras disposiciones, Bogotá, Congreso Nacional de Colombia, <https://www.colciencias.gov. co/node/302> [Consulta: diciembre de 2019].

Congreso Nacional de Colombia (2000), Ley 633 de 2000, Bogotá, Congreso Nacional de Colombia,<https:// www.colciencias.gov.co/node/282> [Consulta: diciembre 2019].
Chompalov, Ivan y Wesley Shrum (1999), "Institutional collaboration in science: a typology of technological practice", Science, Technology, and Human Values, vol. 24, núm. 3, pp. 338-372.

De Haas, Wim (2017), "National research agendas: an international comparison", en Beatrice de Graaf and Alexander Rinnooy Kan (eds.), The Dutch National Research Agenda in perspective: a reflection on research and science policy in practice, Amsterdam, Amsterdam University Press, pp. 47-60.

García Montaña, Amparo (2018), Evaluación de resultados a proyectos del SNR: Sector Ciencia, Tecnología e innovación, Bogotá, Departamento Nacional de Planeación.

Isoglio, Antonela y José Antonio Echeverry-Mejía (2019), "Reconocimiento de experticias y orientación hacia problemas sociales: las prácticas de ciencia abierta desde la perspectiva de la investigación integrada", Ciencia y Sociedad, vol. 44, núm. 1, pp. 29-42.

Kleiche-Dray, Mina y Antonio Chiapa Zanón (2016), "Colaboraciones y construcción de redes de los químicos mexicanos", en Mina Kleiche-Dray y Daniel Villavicencio (eds.), Cooperación, colaboración científica y movilidad internacional en América Latina, Buenos Aires, CLACSO, pp. 161-176.

Latour, Bruno (1987), Ciencia en acción: cómo seguir a los científicos e ingenieros a través de la sociedad, Barcelona, Editorial Labor.

Latour, Bruno (1983), "Give me a laboratory and i will raise the world", en K. Knorr-Cetina y M. Mulkay (eds.), Science observed: perspectives on the social study of science, Londres, Sage, pp. 141-170.

López Turley, Ruth (2016), "Connecting research and policy to reduce inequality", The Russell Sage Foundation Journal of the Social Sciences, vol. 2, núm. 5, pp. 272-285. Montoya, Dolly (2019), "Diálogos en las regiones para armonizar el Sistema de Ciencia, Tecnología e Innovación”, Diario El Espectador, Bogotá, 11 de mayo, <https://www.elespectador.com/opinion/dialogosen-las-regiones-para-armonizar-el-sistema-de-ciencia- 
tecnologia-e-innovacion-columna-860129_blank> [Consulta: enero de 2020].

Nelson, Wendy (2009), "The discipline of rankings: tight coupling and organizational change", American Sociological Reviere, vol. 74, núm. 1, pp. 63-82.

Newman, Kirsty, Catherine Fisher y Louise Shaxson (2012), "Stimulating demand for research evidence: what role for capacity building", IDS Bulletin, vol. 43, núm. 5, pp. 17-24.

Nugroho, Kharisma, Fred Carden y Hans Antlov (2018), "Forms of knowledge and policy influence", en Kharima Nugroho, Fred Carden y Hans Antlov (eds.), Local knowledge matters: power, context and policy making in Indonesia, Bristol, Bristol University Press, pp. 29-42.

Pollitt, Christopher (2020), "Shadowland: the poorlymapped, underdiscussed yet vital interface between public administration research and practice", en Christopher Pollitt (ed.), European perspectives for public administration: the way forward, Lovaina, Leuven University Press, pp. 249-272

Room, Graham (2011), Complexity, institutions and public policy: agile decision-making in a turbulent world, Londres, Edward Elgar Publishing.

Sebastián, Jesús (2000), "Modalidades y tendencias en la cooperación internacional entre las universidades", Revista Española de Desarrollo y Cooperación, núm. 5, pp. 125-144.

Smith, Adrian (2017), "Innovación social, democracia y makerspaces", Revista Española del Tercer Sector, núm. 36, pp. 49-74.

Suarez, Marcela y Gabriela Dutrénit (2014), "The role of innovation policy incentives on the reproduction of asymmetries within nanotechnology knowledge networks in Mexico", Science and Public Policy, <http:// spp.oxfordjournals.org/cgi/reprint/scu005? ijkey=zz HijYoro0jO9lc\&keytype $=$ ref $>$ [Consulta: enero de 2020].

Katz, Silvan y Ben Martin (1997), "What is research collaboration?", Research Policy, vol. 26, núm. 1, pp. 1-18.

Torres Vargas, Arturo (2016), "Intermediación para la transferencia de conocimiento e innovación: el caso de la Fundación Produce de Nuevo León”, en Alexandre Vera-Cruz y Gabriela Dutrénit (eds.), Sistema de innovación del sector agropecuario mexicano: tendiendo puentes entre los actores de la innovación, México, UAM/Miguel Angel Porrúa, pp. 287-312.

Universidad Nacional de Colombia (UNAL) (2020a), Plan de fortalecimiento de la Escuela de Pensamiento de la Universidad Nacional de Colombia, Bogotá, Vicerrectoría de Investigación, UNAL.

UNAL (2020b), Estadísticas, Bogotá, UNAL <http:// planeacion.bogota.unal.edu.co/docentes.html>, [Consulta: octubre de 2020].

UNAL (2019), Informe de Gestión 2019: Investigación, extensión y circulación de saberes, un proyecto cultural y colectivo la nación colombiana, Bogotá, Vicerrectoría de Investigación, UNAL.

Universidad de los Andes (2020), Estadísticas, Bogotá, Uniandes, <https://planeacion.uniandes.edu. co/universidad-en-cifras/universidad-en-cifras $>$ [Consulta: octubre de 2020].

Vessuri, Hebe (2014), "Cambios en las ciencias ante el impacto de la globalización”, Revista de Estudios Sociales, núm. 50, pp. 167-173.

Vessuri, Hebe (2013), “QQuién es el científico social en el siglo XXI?: comentarios desde los contextos académicos y aplicados y desde la corriente principal y la periferia", Sociológica, vol. 28, núm. 79, pp. 201-231.

Cómo citar este artículo:

Medina Muñoz, Lina-Rocío (2021), "El giro de la investigación universitaria hacia la acción pública: el debate sobre los Centros de Pensamiento en Colombia", Revista Iberoamericana de Educación Superior (RIES), vol. XII, núm. 35, pp. 150-168, Dol: https://doi. org/10.22201/iisue.20072872e.2021.35.1087 [Consulta: fecha de última consulta]. 